\title{
Array of Sensors with A/D Conversion Based on Flip-Flops
}

\author{
WEIJIAN LIAN AND SIETSE E. WOUTERS
}

\begin{abstract}
A silicon array of light intensity sensors is described. Switched bias flip-flops are used to obtain a digital output. The symmetry of the flip-flops can be influenced to obtain a method of analogto-digital conversion.
\end{abstract}

\section{INTRODUCTION}

$\mathrm{T}$ HIS PAPER presents an array of silicon flip-flop sensors, such that when the bias of a flip-flop circuit is turned on, their resulting state is very sensitive to the balance of the circuit. A flip-flop, as shown in Fig. 1, has two stable states, "one" and "zero". The flip-flop can be brought into an unstable state by switching the bias current off and, after a short time, switching the bias current back on. During the switch-on period, the flip-flop is in the unstable state. Once it is on, it will switch to either a "one" or a "zero". The direction of switching can be influenced by external parameters. For instance, the transistors in the flip-flop can be designed to be sensitive to light. When only one of the two transistors is illuminated, an asymmetry will be created and the flip-flop will switch to a given stable state.

The process in which a flip-flop is brought into an unstable state, with the subsequent switching to a stable state, is repeated periodically with a high frequency. The number of the resulting "ones" is counted and this is a digital measure of the external parameter [1]. The flipflop sensor has the following features: a) a digital output is realized; b) a high threshold-sensitivity, which is defined as the smallest quantity that can be detected, is obtained; c) the system has a simple structure, and d) the flip-flop sensor is suitable for the construction of large sensor arrays.

By virtue of the small size of the flip-flop sensor, a large number of them can be integrated in an array. The device described further in this paper consists of optical sensors. Each sensor consists of two resistors and two silicon phototransistors, one of which is covered by aluminum in order to allow the light to generate an asymmetry in the flipflop. In an early experimental device [2], the sensors were designed to have a fixed threshold level determined by the ratio of the flip-flop load resistances. The new device presented here has an electrically controllable threshold. The

Manuscript received August 1, 1989; revised February 22, 1990

The authors are with the Department of Electrical Engineering, Delft University of Technology, GA Delft, The Netherlands.

IEEE Log Number 9036020.

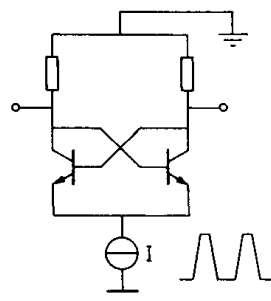

Fig. 1. Circuit diagram of a flip-flop.

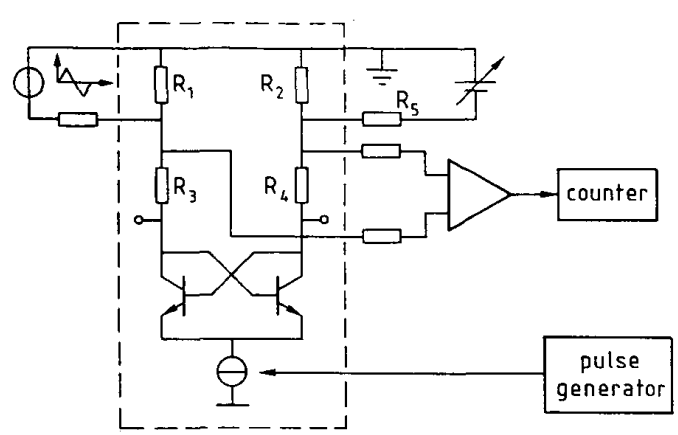

Fig. 2. A triangle wave voltage is added to the flip-flop.

two sides of a flip-flop in the new device are biased by two separate voltages as shown in Fig. 2, making it possible to control the threshold level by a triangle-wave voltage in order to realize $A / D$ conversion for a large input range.

\section{Circuitry and Operation of the Array}

\section{A. Flip-Flop Sensors}

Phototransistors, photodiodes, thermocouples, temperature sensitive transistors, Hall plates, nMOS split-drain magnetic transistors and piezoresistors can all be implemented in a flip-flop sensor array. In order to integrate a large matrix of individual fiip-flop sensors onto one wafer, it is important that each flip-flop efficiently utilizes only a small chip area. Therefore, the most suitable flip-flop sensors for array construction are those which use the components of the flip-flop-resistors and transistors-as the sensing elements.

The flip-flops can be positioned in a matrix. Flip-flops of the same row would share the same bias current, as shown in Fig. 3. This structure would automatically ensure that the currents passing through each flip-flop would 


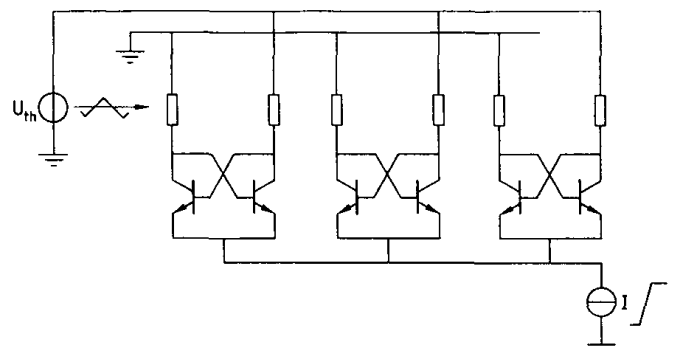

Fig. 3. Circuit diagram demonstrating a row of flip-flops. The resistors on the left are connected to ground. The others are connected to the triangle wave voltage $U_{\mathrm{th}} . I$ is the bias current, which can be switched on/off.

be the same because the voltages over each flip-flop would be the same.

A significant difference between a flip-flop sensor and a flip-flop sensor array is that a flip-flop sensor has only one offset, which can be compensated by one dc voltage. However, in a flip-flop sensor array each flip-flop would have its own offset, which therefore would demand a large number of compensation voltages. The use of one slow (for instance $1 \mathrm{~Hz}$ ) triangle wave voltage for the whole array can simulate all the necessary dc voltages. In effect, the triangle wave would scan a certain range within which all offset values would lie, and thus would provide each flip-flop with the opportunity to compensate its offset. The triangle wave must be slow in comparison with the rates at which the flip-flops are brought into an unstable state.

Fig. 3 illustrates the implementation of the triangle wave voltage. All flip-flops have one of their load resistors connected to the ground and the other connected to the triangle wave voltage. The individual offset of each flip-flop must be measured and the results must be stored in the memory of a computer. The offset must then be subtracted by a computer in order to obtain a result.

\section{B. Selection and Bias Circuitry}

Current sources are used to provide the bias currents for the flip-flops. Current switches are used to perform the row and column selection. These circuits are very simple and will not be discussed here in detail.

\section{Flip-Flop Sensor Array}

The electrical schematic of the array is illustrated in Fig. 4, which shows a $2 \times 2$ array. $U_{\text {th }}$ is the triangle wave voltage to which the right sides of all flip-flops are connected; the left sides are connected to ground. $U_{\text {stb }}$ controls the on/off switching of the flip-flops and supplies the standby currents.

The triangle wave voltage $U_{\text {th }}$ changes the threshold of the flip-flops slowly (low frequency) while $U_{\text {stb }}$ switches the flip-flops on, thus bringing them into an unstable state and completing the sense action. Then the signals $U_{\text {sell }}$ and $U_{\text {sel2 }}$ determine which row is read out. After read out, $U_{\text {stb }}$ switches off the flip-flops for reset before switching them on again and during this time $U_{\text {th }}$ has taken the next value.

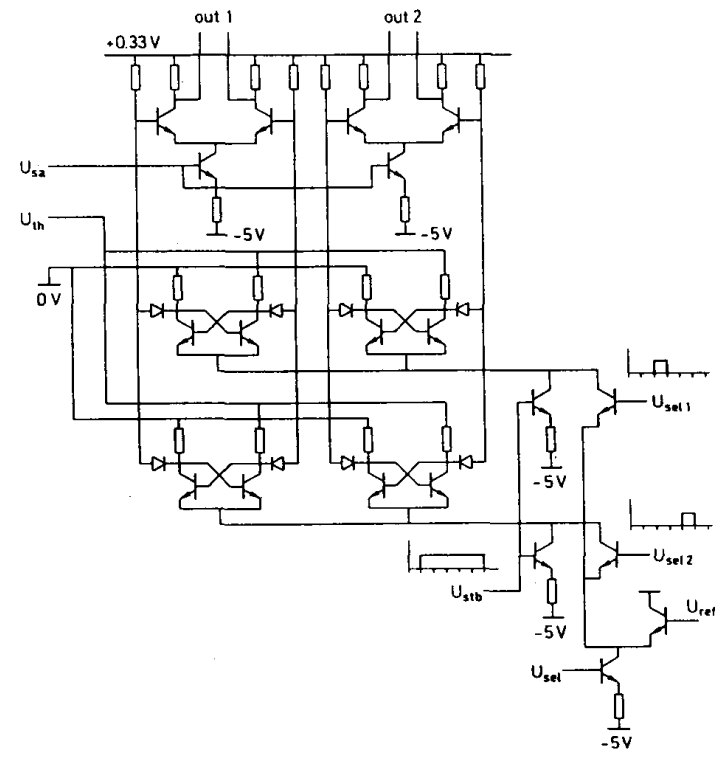

Fig. 4. Circuit diagram demonstrating 4 flip-flop sensors, two sense amplifiers and the necessary selection and bias circuitry. $U_{\mathrm{th}}$ is the triangle wave voltage.

There are several ways to operate the array. After each switch-on pulse of $U_{\mathrm{stb}}$, only one flip-flop sensor (for instance the upper left one in Fig. 4) was read out. Therefore, after one period of the triangle wave, only that one flip-flop measurement was completed. Then, in the second period of the triangle wave the next flip-flop measurement was carried out. The number of periods of the triangle wave necessary to complete measurements for the whole array was equal to the number of flip-flops in the array. However, the duration of the periods of the triangle wave can be shorter, because after each unstable state only one flip-flop needs to be read out. This can, to some extent, compensate the effect of the larger number of periods of the triangle wave on the total measurement time. The advantage of this method is the simplicity. Because only one flip-flop is active at one time, only one counter is needed to count the number of "ones".

Another mode of operation is to read out a number of flip-flops simultaneously after an unstable state. This can be done by using one counter for one column of the array. Therefore, in one period of the triangle wave, one row of the array was measured. Then, in the next period the next row was measured. Such a parallel operation greatly increases the speed of measurements. The disadvantage is that more than one counter is necessary.

Finally, all flip-fiops can be read out after each unstable state. In this way, only one period of the triangle wave is needed, thus giving this method the potential to be the fastest. However, the counting process will be rather complex and can only be performed through the control of microprocessors.

These operating modes show the flexibility of the device. The mode of operation employed has no conse- 
quence on the design, as the mode most suitable depends on the specific application.

\section{Resolution of the A/D Conversion}

This section presents an analysis of the resolution of the measurement technique when used for low-signal level high-resolution measurements.

The measurement process is illustrated in Fig. 5. When the magnitude of the triangle wave is lower than the signal $s$, the output of the flip-flop will be "zero". When the magnitude of the triangle wave is higher than the signal $s$, the output will be "one". The total number of "ones" counted during one period of the triangle wave is used as the output. If the offset is zero, the output is then $N_{1}=$ $\left(s+V^{+}\right) N /\left(2 V^{+}\right)$where $V^{+}$is the amplitude of the triangle wave voltage, $s$ is the equivalent signal voltage, and $N$ is the number of unstable states during one period of the triangle wave. The offset effectively adds a constant number to $N_{1}$. Therefore, in practice, this constant number must be subtracted from $N_{1}$.

The measurement error discussed here is caused by the random noise. In fact, the threshold level of the flip-flop is subjected to random noise. Fig. 5(c) and Fig. 5(d) illustrate this situation. When the magnitude of the triangle wave is lower than the signal, the output has to be a "zero", but because of noise it can be a "one". As a result, $N_{1}$ is not constant but is subjected to random changes. The standard deviation of such random changes is defined here as the error of the measurement.

The larger the number of unstable states taken within a certain measurement range, the smaller the error. This error depends on the noise voltage, the number of unstable states, and the amplitude of the triangle wave voltage. The relation between the error on the one side together with the noise voltage, the number of unstable states, and the amplitude of the triangle wave on the other side is derived in this section. In fact, only the upper limit of the error is presented. The exact value of the error can be calculated by only using complex numerical methods and is not discussed here.

A detailed description of how the error occurs can be given using the illustration of Fig. 6. The $\mathrm{x}$-axis represents the differential voltage consisting of $U_{\text {th }}-s$, where $U_{\text {th }}$ is the triangle wave and $s$ the signal. In the ideal case (zero noise) when $U_{\text {th }}$ is lower than $s U_{\text {th }}-s$ and is negative, the output is a "zero"' as shown by " $\downarrow$ "' in Fig. 6. When $U_{\text {th }}-s$ is positive, the output is a "one". Because of noise, the output can be a "one" even though $U_{\text {th }}-s$ is negative. The curve in Fig. 6 shows the probability of obtaining a "one"' against $U_{\text {th }}-s$.

For $\left|U_{\text {th }}-s\right| \geq 3 u_{\mathrm{n}}$, where $u_{\mathrm{n}}$ is the noise voltage, the probability of obtaining an incorrect output is negligible. Thus only those unstable states located within the range $\left|U_{\text {th }}-s\right|<3 u_{\mathrm{n}}$ are considered. The number of "ones" within this range, on the average, is equal to half the number of unstable states in that range due to the symmetry of Fig. 6. The error is thus the standard deviation of the number of "ones"'. The closer to the origin of Fig.
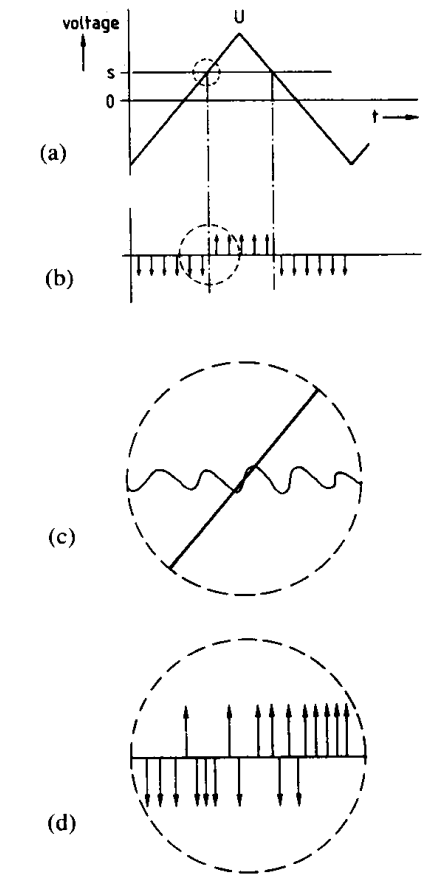

Fig. 5. (a) The two parameters $s$ and $U_{\mathrm{th}}$, which determine the final stable states of the flip-flop sensors. (b) When $U_{\text {th }}$ is lower than $s$, the outputs are "zeros"; when $U_{\mathrm{th}}$ is higher than $s$, the outputs are "ones". At the point $s \approx U_{\mathrm{th}}$, (transition point) marked by the $c^{\circ} \cdot \therefore$ errors can occur. $\begin{array}{lll}\text { (c) The cause of the errors, namely the random } n & \text { i) Flip-flop sensor }\end{array}$ output with errors.

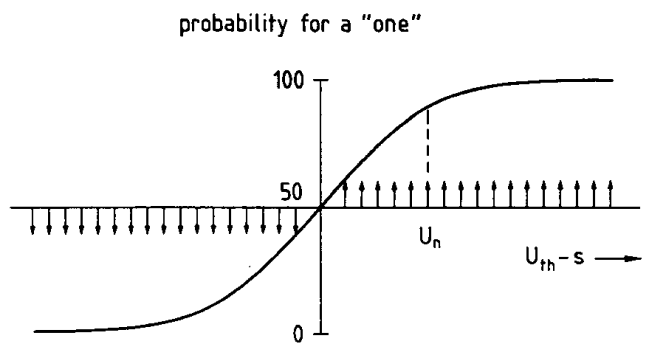

Fig. 6. Probability ( $y$-axis in \%) of obtaining a "one," versus the differential signal ( $x$-axis, normalized to the noise voltage) $U_{\mathrm{th}}-s$. The array of " $\uparrow$ " and " $\downarrow$ " represents the series of "ones" and "zeros" in an imaginary error free situation. In reality the transition from "one" to "zero" changes gradually. The maximum error occurs at $U_{\mathrm{th}}=s=0$.

6 the unstable state is located, the larger the probability is of obtaining an incorrect output. The maximum probability of obtaining an incorrect output appears at the origin and is equal to $50 \%$. An upper limit of the error can thus be derived by assuming that all unstable states have the maximum probability of $50 \%$.

The problem is then simplified. For an amplitude of $V^{+}$ for the triangle wave in Fig. 5 and for the number of unstable states in one period equal to $N$, the voltage change of the triangle wave between two unstable states is 2 $V^{+} /(N / 2)$. If $N_{\mathrm{u}}$ is the number of unstable states taken within the range of $\left|U_{\mathrm{th}}-s\right| \leq 3 u_{\mathrm{n}}$, then $N_{\mathrm{u}}$ is equal to 
$2 \times 3 u_{\mathrm{n}} /\left[2 V^{+} /(N / 2)\right]$. If each unstable state has a $50 \%$ chance of obtaining a "one", what is then the standard deviation in the counted number of "ones"? The answer to this question is given by

$$
\begin{aligned}
N^{\prime} & =\sqrt{0.5 \times 0.5 N_{\mathrm{u}}} \\
& =\sqrt{0.5 \times 0.5 \times 2 \times 3 u_{\mathrm{n}} /\left[2 V^{+} /(N / 2)\right]} \\
& =\sqrt{0.5 \times 0.5 N 3 u_{\mathrm{n}} /\left(2 V^{+}\right)}
\end{aligned}
$$

where $N^{\prime}$ is the standard deviation in the number of "ones",

In one period of the triangle wave, the situation in which $U_{\text {th }}$ crosses $s$ occurs twice and this causes the actual standard deviation is reduced by $\sqrt{2}$. The total error $N_{\mathrm{e}}$ is thus

$$
\begin{aligned}
N_{\mathrm{e}} & =\sqrt{0.5 \times 0.5 N_{\mathrm{u}} / 2} \\
& =\sqrt{0.5 \times 0.5 N 3 u_{\mathrm{n}} /\left(4 V^{+}\right)} \\
& =\sqrt{N 3 u_{\mathrm{n}} /\left(16 V^{+}\right)} .
\end{aligned}
$$

The voltage $u_{\mathrm{e}}$ equivalent to this number is given by

$$
u_{\mathrm{e}}=N_{\mathrm{e}} 2 V^{+} /(N / 2)=\sqrt{3 u_{\mathrm{n}} V^{+} / N} .
$$

The above equations for $N_{\mathrm{e}}$ and $u_{\mathrm{e}}$ present the relation between the error on the one side and the noise, triangle wave amplitude and number of unstable states on the other side. For example, when the noise is $20 \mu \mathrm{V}$, the amplitude is $20 \mathrm{mV}$ and the desired error is $1 \mu \mathrm{V}$, how many unstable states should be taken? Using the relation for $u_{\mathrm{c}}$, the answer is found to be $N=12 \times 10^{5}$.

The above analysis is suitable for measurements in which the signal level is low and the desired accuracy is high. When the signal level is high and a lower accuracy is sufficient, the number of unstable states $N$ can be smaller. If $N$ is so low that the change in the triangle wave voltage between two unstable states is larger than the noise voltage, then the error is determined by the quantization error and is equal to $N_{\mathrm{e}}=2 V^{+} / N$.

\section{Experimental Results}

Optical flip-flop sensor arrays based on phototransistors were fabricated in the bipolar process. The devices contain 64 flip-flop sensors organized in $8 \times 8$ arrays. Each flip-flop sensor consists of two resistors and two phototransistors, of which one is covered by aluminum. The design specifications utilized are given below.

1) A flip-flop sensor size of approximately $250 \times 250$ $\mu \mathrm{m}^{2}$ was used. This area is close to the minimum size, which is determined by the bipolar technology design rules.

2) The flip-flop effective sensing area is $3620 \mu \mathrm{m}^{2}$. The phototransistor, which is supposed to be covered by aluminum, is not completely covered. There is a small percentage of sensing area left open, due to the necessary spacing between two separate aluminum lines. The effective sensing area is then the difference of the sensing areas of the two phototransistors.

3) Each load resistance of the flip-flop sensors is

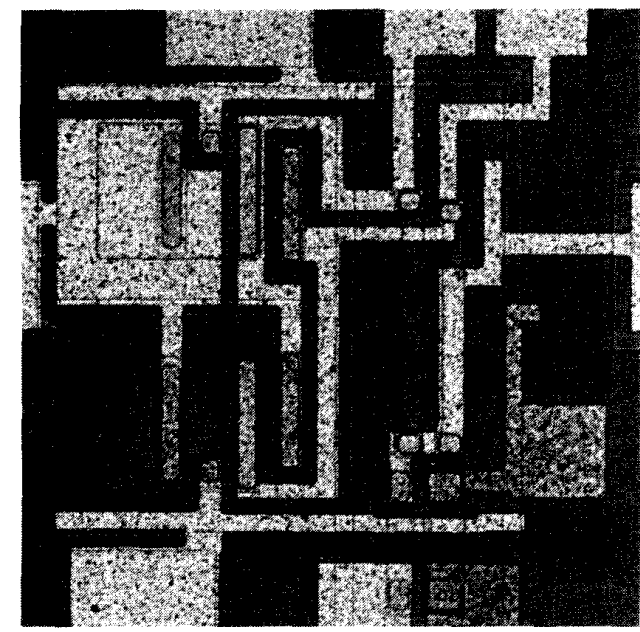

Fig. 7. Microphotograph of a phototransistor flip-flop sensor.

$20 \mathrm{k} \Omega$. This value has influence on the speed and sensitivity of the flip-flop sensors. The resistors were made using a boron implantation, which provides accurately matched resistors and a high sheet resistance of $2 \mathrm{k} \Omega / \square$.

4) The total area of the chip containing flip-flops, sense amplifiers, selection and bias circuitry is $3 \times 3 \mathrm{~mm}^{2}$.

Extreme care was taken in the design of the layout to minimize the flip-flop area. The fabrication technology determines the area. Efficient design of the layout also has a large influence. The positions and orientation of the transistor collectors, bases and emitters and the resistors must be carefully considered. The undercrossings require ample space and thus require much attention. The diodes connected to the sense amplifiers were integrated in the same island as the transistors. A separate p-type diffusion area fabricated using the base diffusion was used as the $\mathrm{p}$-region of the $\mathrm{p}$ - $\mathrm{n}$ diode. The $\mathrm{n}$-region is then the island. Such a construction yields a very compact device. Fig. 7 shows a microphotograph of a phototransistor flip-flop sensor. The devices were tested using a microprocessor controlled system as shown in Fig. 8. The computer controlled the amplitude, the frequency and the starting-point of the triangle wave. The IEEE Interface was used to match the signal levels of the array and the computer. The computer generated the selection signals and read out the results of the array.

The counting was performed by the computer. To each flip-flop, a memory location in the computer was assigned at which the number of "ones" was stored. After an unstable state, the computer decided whether or not to increase the number of "ones", depending on a "one" or a "zero" output. The offset was also stored in the computer and was subtracted from the measured number of "ones".

The flip-flop sensor sensitivity to light intensity is measured for a light wavelength of $638 \mathrm{~nm}$. On the average, $0.26 \mathrm{mV}$ output voltage is generated by a light intensity of $1 \mathrm{klx}\left(\operatorname{lux}=6.21 \times 10^{2} \mathrm{~W} / \mathrm{cm}^{2}\right)$, for a sensor with a 


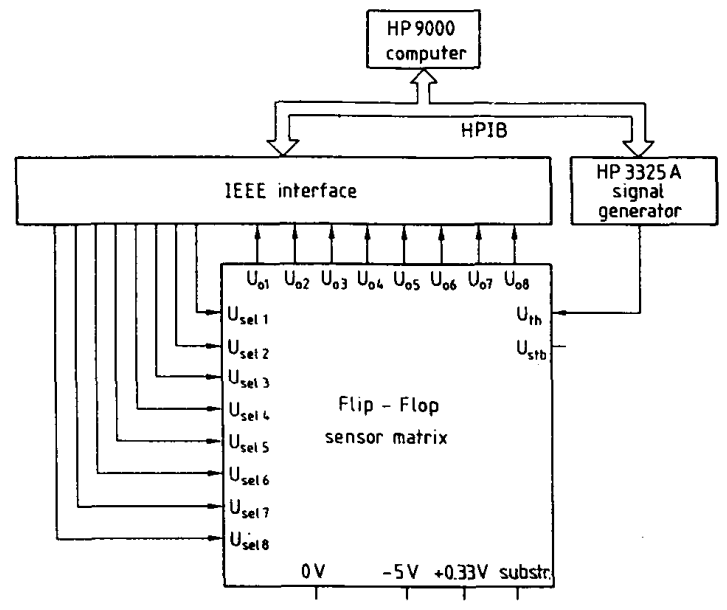

Fig. 8. Diagram of the computer controlled measurement system.

sensitive area of $3620 \mu \mathrm{m}^{2}$. The speed of individual flipflop sensors reached $5 \mathrm{MHz}$. This speed is determined by the load resistance and the cutoff frequency of the phototransistors, which was $350 \mathrm{MHz}$. At this speed, SPICE simulation showed that, provided the measurement setup allowed such a frequency, the whole array can operate at a frequency of $625 \mathrm{kHz}$. The offset, which is determined by the mismatches, is dependent on the fabrication process. Different batches have different offset values. When the fabrication process is successfully performed, the offset will range from $0.1 \mathrm{mV}$ to several millivolts and it occasionally will exceed $10 \mathrm{mV}$. Although the offset is subtracted from the measurement results, a comparatively small offset is still very favorable in practice because comparatively small offsets require lower triangle wave amplitudes and yield a smaller change in the offset values when temperature changes. The noise of the flip-flop sensor is measured to be $50 \mu \mathrm{V}$.

\section{CONClusion}

A silicon light-intensity sensor array is made using switched bias flip-flops. The flip-flops contain bipolar transistors which are sensitive to illumination. A trianglewave voltage is used for analog to digital conversion. Experiments showed that the principle works. The quality of the device depends on the processing. Offset voltages of the flip-flops in the array ranged from $0.1 \mathrm{mV}$ to several millivolts.

\section{REFERENCES}

[1] W. Lian and S. Middelhoek, "A new class of integrated sensors with digital output based upon the use of a flip-flop," IEEE Electron Device Lett., vol. EDL-7, pp. 238-240, 1986.

[2] W. Lian, P. J. French, and Y. Z. Xing, "Pattern detectors based upon flip-flop sensor arrays," in 4th Int. Conf. on Solid-State Sensors and Actuators, Tokyo, Japan, pp, 143-146, June 2-5, 1987

[3] S. E. Wouters and W. Lian, "A flip-flop sensor array with on-spot A/D conversion," in I4th European Solid-State Circuits Conf., pp. 335-338, Manchester, UK, Sept. 21-23, 1988. 